\title{
Maintaining Continuity in Longitudinal, Multi-Method Health Interventions Using Virtual Agents: The Case of Breastfeeding Promotion
}

\author{
Zhe Zhang ${ }^{1}$, Timothy Bickmore ${ }^{1}$, Krissy Mainello ${ }^{2}$, Meghan Mueller ${ }^{3}$, Mary Foley ${ }^{4}$, \\ Lucia Jenkins $^{5}$, Roger A. Edwards ${ }^{2}$ \\ ${ }^{1}$ College of Computer and Information Science, Northeastern University, Boston, MA, USA \\ \{zessiez, bickmore\}@ ccs.neu.edu \\ ${ }^{2}$ Bouvé College of Health Sciences, Northeastern University, Boston, MA, USA \\ \{k.mainello, ro.edwards\}@ neu.edu \\ ${ }^{3}$ Northeastern University, Boston, MA, USA \\ \{meghanlmueller\}@gmail.com \\ ${ }^{4}$ Melrose Wakefield Hospital, Hallmark Health, Melrose, MA, USA \\ \{mfoley\}@ hallmarkhealth.org \\ ${ }^{5}$ Baby Café USA, Wakefield, MA, USA \\ \{luciansla\}@aol.com
}

\begin{abstract}
Virtual agents can provide a sense of continuity in applications that span long periods of time and incorporate diverse activities, media, and modalities. We describe the design of a virtual lactation educator - agent that promotes breastfeeding in three settings, across different time spans, using a range of media and counseling techniques. The agent provides "interpersonal continuity of care" that is important in many areas of medicine. The results of a pilot study and an ongoing clinical trial are presented.
\end{abstract}

Keywords: Relational agent, embodied conversational agent, breastfeeding, medical informatics, health informatics.

\section{Introduction}

Many health interventions require multiple contacts with users over extended periods of time to be effective. Health behavior change interventions, such as walking promotion or smoking cessation, can often take months or years of sustained coaching to succeed, even when the behaviors involved are relatively simple. Some health interventions, however, require not only sustained effort over time but the use of a wide range of counseling and instructional techniques, media, and intervention modalities. For example, the promotion of self-care management for newly-diagnosed individuals with diabetes typically requires education about the disease, instruction in selfinspection techniques (foot exams to prevent ulcers resulting from diabetic neuropathy), and longitudinal behavior change counseling on diet, exercise, and medication adherence. Such longitudinal, multi-method and multi-modal interventions are partic- 
ularly challenging to automate, because of the range of topics to be covered, the range of pedagogical and persuasive techniques that must be leveraged, the changing needs of users over time, and the need to maintain user engagement over the months or years required.

Breastfeeding promotion is another example of a health behavior that requires such a longitudinal multi-faceted intervention [1]. The intervention should start prenatally by educating women about the advantages of breastfeeding, motivating them to begin breastfeeding immediately after birth, and providing them with basic information about how to breastfeed and where to find additional information. Immediately following the birth of their babies, women need critical information about how to get started breastfeeding, help troubleshooting common problems, and the means for contacting a lactation consultant should significant problems arise. Once they transition home, women need information on a wide range of topics related to breastfeeding, sustained motivation for continuing breastfeeding in the face of common obstacles, help tracking their behavior and outcomes, and a mechanism for alerting clinicians if significant problems arise. Each of these elements may be best delivered through a different type of media, from text and images, dialogue, and graphs to video clips demonstrating different breastfeeding techniques.

Virtual agents may be a particularly well-suited medium for delivering such complex interventions, given their ability to provide a persistent, continuous presence across computing platforms, across time, and across different intervention modalities. In addition to functioning as a breastfeeding counselor, an agent can also play the role of instructor, cheerleader, and confidant, and use a wide range of media in its counseling sessions with users, while maintaining the user-agent dialogue and relationship as an anchor during the months-long intervention. In medicine, such "interpersonal continuity of care" across visits has been shown to be a significant determinant of patient satisfaction and health outcomes [2].

In this paper we describe our experience developing a virtual agent that promotes breastfeeding from the third trimester to six months after birth, across a range of computing platforms and use sites, and using a range of pedagogical and motivational techniques and media. We first describe the importance of breastfeeding, and previous work in automating breastfeeding promotion, before discussing our development methodology, and the design of our agent and intervention. We then present the results of a pilot study and preliminary results from an ongoing clinical trial before concluding.

\section{The Importance of Breastfeeding}

There are many health benefits of breastfeeding for both mothers and babies. According to the American Academy of Pediatrics, infants who receive any breast-feeding experience $23 \%$ lower risk of inflammation of the middle ear, $64 \%$ lower risk of gastroenteritis, $40 \%$ lower risk of Type 2 diabetes, $31 \%$ lower risk of inflammatory bowel disease as well as a $15-30 \%$ reduction of adolescent and adult obesity rates in infants who were breastfed. In addition, a 35\% lower risk of SIDS is found in infants 
who receive any breastfeeding longer than 1 month. Additional health benefits accrue when intensity and duration of breast-feeding increases. For example, infants who are exclusively breastfed for the first 3 months of life have a $42 \%$ lower risk of developing atopic dermatitis, an inflammatory skin disorder, as well as a $30 \%$ reduction in risk of developing Type 1 diabetes and a $40 \%$ lower risk of asthma when the atopic family history is positive ( $26 \%$ when family history is negative). Breastfeeding at 6 months results in a $63 \%$ lower risk of upper respiratory tract infection and a $20 \%$ lower risk in childhood leukemia [22].

There are also numerous health benefits to mothers who breastfeed their infants. For example, breastfeeding moms have less postpartum bleeding, experience lower rates of postpartum depression and have lower rates of breast and ovarian cancers [22]. Although evidence remains inconclusive, there are studies that have shown women who breastfeed return to pre-pregnancy weight faster than moms who do not breastfeed their newborns. In addition, women who have breastfed for a cumulative period of 12-23 months have a significant reduction in hypertension, hyperlipidemia, cardiovascular disease and diabetes [3].

For maximum health benefits, the current World Health Organization (WHO) and American Academy of Pediatrics (AAP) recommend that women breastfeed their infants exclusively for the first 6 months of life and up to 1 year or longer if mutually desired by the mother and child. Although there have been several initiatives aimed at improving exclusive breastfeeding rates in the US, including the Healthy People national disease prevention goals in 2000, 2010 and 2020 and a 2011 Surgeon General's Call to Action to Support Breastfeeding, a significant disparity in breastfeeding rates persists in the US. In 2013 the percentage of women who reached the current AAP and WHO recommendation was only $16.4 \%$ nationwide [4].

\section{$3 \quad$ Related Work}

Several virtual agents have now been developed to support longitudinal interactions with users, and several studies on longitudinal effects and continuity have been conducted. Many of the longitudinal applications have been in the health domain $[5,6$, 7], including interventions for women's preconception health [5], but also in gameplaying and education [8]. Studies have explored methods for maintaining longitudinal engagement [23], and assessing the sense of continuity when an agent changes form or moves from one body to another [24].

Several hundred non-automated breastfeeding interventions have now been evaluated, with generally positive outcomes [9]. A few automated systems have been developed to promote breastfeeding among mothers. Most of the interventions developed to date have been educational materials deployed on static web pages or multimedia CD-ROMs ([10, 11]). Joshi, et al, describe a bilingual touch-screen tabletbased intervention to promote breastfeeding. However, the system is not a longitudinal intervention (designed for single contact use), and has not been evaluated in a clinical trial (only formative usability testing results are reported) [12]. Emrick describes the development of "Latch Master", an iPhone game designed to teach moth- 
ers about correct breastfeeding positions and latching, although no evaluation is reported [13].

\section{Design of a Virtual Agent for Breastfeeding Promotion}

The primary support for breastfeeding mothers is face-to-face counseling along with paper-based information materials. However, the effectiveness of such support is limited by several major issues. Professionals are not always able to spend a sufficient amount of time with each mother going through necessary information. Lack of conformity in recommended guidelines and advice also impedes mothers' perception of high-quality information. By using virtual agents, an entire consultation can be virtually simulated where mothers are free to spend as much time as needed interacting with the agents to absorb knowledge and resolve concerns. We developed a virtual agent to promote and support breastfeeding among new mothers. The agent is designed to interact with mothers at three time points:

1. Prenatally, during the third trimester, typically in the obstetrician's office, on a tablet computer. The overall objective of this module of the intervention is to motivate women to choose to breastfeed-by emphasizing the health benefits for the baby and the mother - and to provide information on what to expect and how to get started with the initial breastfeeding attempts.

2. Perinatally, immediately following birth, in woman's room in the maternity unit of the hospital, either on a wheeled computer kiosk or a tablet computer. The objective of this module of the intervention is to provide "breastfeeding 101 " information on how to get started, to provide initial tracking capability (number of feeds, number of soiled diapers), and to provide referral to a human lactation consultant if needed.

3. Postnatally, once a woman leaves the hospital and returns home, for the following six months, accessed daily over the web. This overall objective of this module is to promote adherence to the CDC-recommended six months of exclusive breastfeeding, and to provide women with time-based information needed by breastfeeding mothers.

The virtual agent was developed to play the role of a virtual lactation educator. Our development methodology involved initially videotaping sample counseling sessions with an International Board Certified Lactation Consultant. This was followed by several months of meetings of the interdisciplinary team to work through the overall design of the system and the specific dialogue scripts and media content used in each part of the intervention.

The virtual agent's appearance was designed based on feedback from user testing with new mothers (Figure 1). The agent is rendered in a web-based Unity plugin, using the LiteBody framework [14], with speech output produced on the server with a commercial speech synthesizer. Dialogues are scripted using a custom hierarchical 
transition network-based scripting language. Agent nonverbal conversational behavior is generated using BEAT [15], and includes beat (baton) hand gestures and eyebrow raises for emphasis, gaze away behavior for signaling turn-taking, and posture shifts to mark topic boundaries, synchronized with speech. User input is obtained via multiple choice selection of utterances [16].
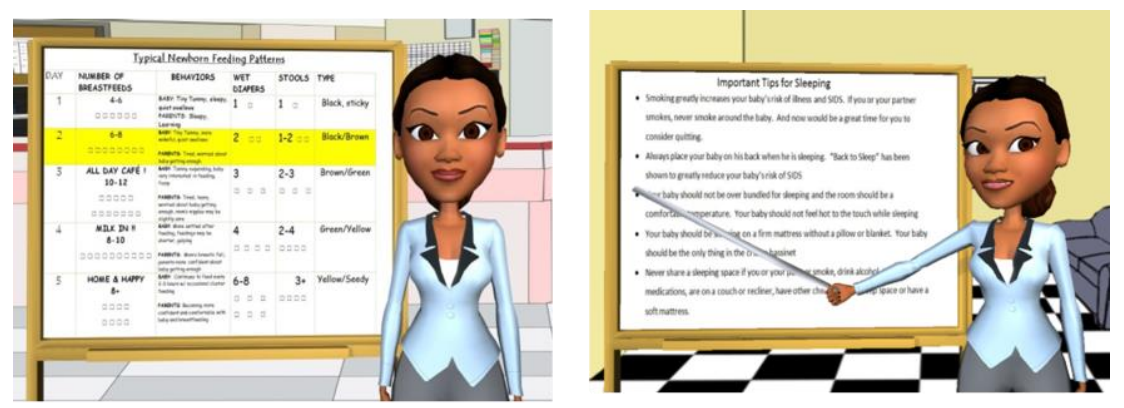

Figure 1. Virtual Breastfeeding Promotion Agent

\subsection{Intervention Methods, Modalities, and Media}

A wide range of intervention methods and media is used in the agent-based breastfeeding intervention, including:

Agent Dialogue. Conversation with the agent is the primary communication modality used in the system, and the overall intervention is framed as a series of conversations with a virtual lactation consultant. The agent uses a number of counseling techniques to educate and motivate the user, including techniques from Motivational Interviewing to motivate [17], and social cognitive techniques to maintain adherence (goal setting, positive reinforcement, problem solving). In addition to therapeutic dialogue, social dialogue and empathy were used to establish rapport and therapeutic alliance with the agent to increase adherence [18]. Additional media were typically introduced to support dialogue (Figure 1).

Educational Content. All three interventions used text and images to provide additional educational content, outlines of topics under discussion, and digital versions of paper forms the agent could explain to the user (Figure 1).

Demonstration Videos. A variety of short video clips were used in the system to demonstrate different breastfeeding positions, hand expression, the "birth crawl" and other physical behaviors that would be very difficult to describe in speech and static images.

Longitudinal Tracking. The perinatal system helps women track the number of breastmilk feedings and soiled diapers to gage their baby's progress while in the hospital. The postnatal system tracks the baby's weight and plots it against norms so that the mother can be reassured that their infant is getting enough nourishment (Figure 2) Significantly underweight babies are automatically reported to study personnel for follow-up. 
Time-based Content. The postnatal module uses a complex schedule of topics that the agent introduces to mothers based on the age of their infant (Figure 3). Topics are designed and delivered daily for the first two weeks, weekly from the third week to the eighth week, and monthly from the third month towards the end of the six-month intervention. Since we cannot control how frequently mothers access the system, strategies were devised for "timing out" of topics that may no longer be relevant, and prioritization among multiple accrued topics.

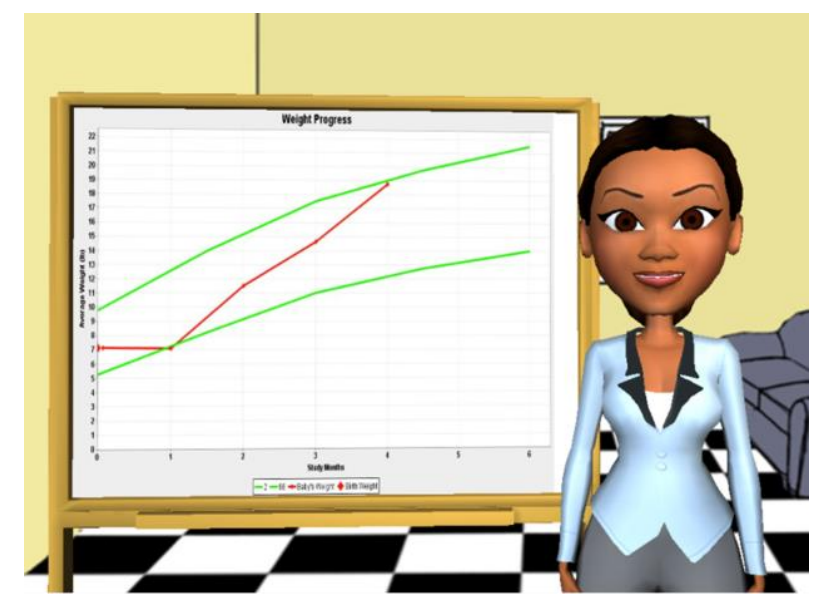

Figure 2. Agent Showing Infant Weight Chart

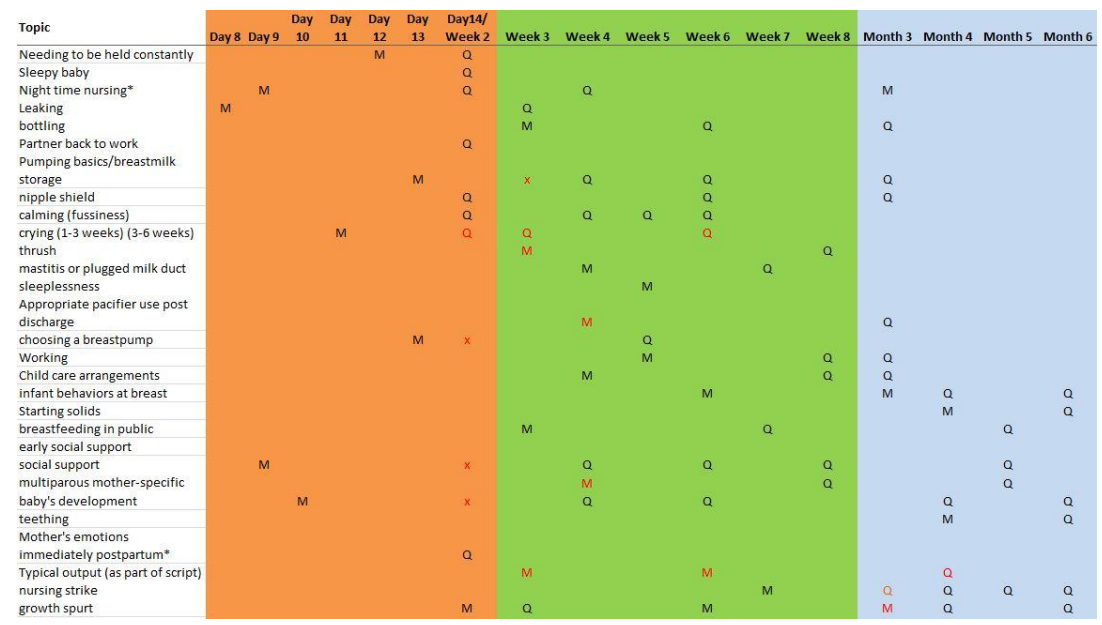

Figure 3 Excerpt of Educational Breastfeeding Topic Schedule after the first week of the six-month intervention $\mathrm{M}=$ Mandatory topic (user must hear, otherwise offered as optional); $\mathrm{c} / \mathrm{s}=\mathrm{C}$-section delivery (vs. vaginal)

Adaptive Interactions. The postnatal module is designed to transition mothers from their hospitalization to their postnatal care. The interactive content is adapted based on a mother's personal progress and her prior interactions with the agent. To avoid 
overwhelming users, we only focus on two mandatory topics in each interaction but allow flexibility for users to be able to review other optional topics.

Homework Assignments. The postnatal module also integrates a certain number of homework assignments that were designed to help the mother obtain support in the community. The agent plays a role to convey the importance of the community support in breastfeeding behavior and introduce the related assignments to mothers.

Interpersonal Continuity of Care. Finally, all three modules use a common database that contains all of a user's information in order to support continuity of care across the nine months of end-to-end system use. A clinician interface allows study personnel to update the database with new information, such as the date and time of a birth, a baby's name and gender, and whether the delivery was vaginal or C-section. Most importantly, we used the same virtual agent character in all three systems, along with dialogue to refer back to prior conversations, in order to provide users with the perception of interpersonal continuity of care.

\section{$5 \quad$ Pilot Evaluation Study}

We conducted a pilot evaluation study of the first two modules with fifteen women who delivered at Melrose-Wakefield Hospital in Massachusetts (previously reported in [19]). Participants—aged 27.9 (sd 5.7), 80\% white-were randomized to the agentbased intervention or standard care. Overall satisfaction with the agent was rated 5.7 on a 7-point scale ( $1=$ not at all satisfied, $7=$ very satisfied). On a 7-point scale assessing subjects' overall confidence in the agent's ability to help, mean scores were 5.9 for the prenatal module and 6.7 for the perinatal module. Those in the intervention group demonstrated significantly greater intent to exclusively breastfeed following their interaction with the prenatal module $(\mathrm{p}<.05)$, and significantly greater breastfeeding knowledge following interaction with the perinatal module $(\mathrm{p}<.05)$, compared to the control group.

\section{$6 \quad$ Ongoing Clinical Trial}

We are currently conducting a randomized controlled trial to evaluate all three modules of the breastfeeding promotion agent. As in the pilot, we are comparing women who interact with the agent to a second group randomized to receive standard care at Melrose-Wakefield Hospital in terms of their initiation and duration of exclusive breastfeeding. We are also interested to explore the impact of the intervention on mothers' attitude towards breastfeeding and their confidence in breastfeeding.

\subsection{Measures}

Self-report measures are administered at the end of the first two weeks after delivery, at the end of two months, and at the end of six months. Confidence in breastfeeding is measured for both intervention group and control group using the 14-item Breastfeed- 
ing Self-Efficacy Scale Short-Form (BSES-SF) [20]. Intervention participants also complete two additional questionnaires about their satisfaction with the Agent and their attitude towards the Agent, assessed using a modified version of the Working Alliance Inventory [21]. Intervention participants' use of all three modules is assessed from database and log file analysis.

The system also enables tracking of when a breastfeeding mother starts to supplement and why. This information will provide the basis for subsequent generations of the agent and what content might be needed to help her continue to breastfeed.

\subsection{Procedure}

Participants are recruited through Melrose-Wakefield Hospital and peripheral obstetrics-gynecology offices. Women were required to be pregnant and in the third trimester either with their first baby or a subsequent baby if they did not reach their breastfeeding goals with their previous children. Women were excluded if they indicated frequent use of alcohol during pregnancy, use of street drugs, pregnant with twins or more and known medical complications or high-risk pregnancies.

A particular challenge in this study is providing a computer-based intervention to patients in the hospital given that we do not know what day they will arrive for delivery. Study staff in the hospital monitored Labor and Delivery to determine when enrolled patients were admitted in labor. When a subject participating in the study was identified as being admitted, study staff visited them on the birth day of her baby to complete questionnaires and provide access to the Agent. Study staff are monitoring use and following-up with mothers if any "red flags" are noted. Institutional Review Board approval was obtained from both Northeastern University and MWH. All subjects provided the informed consent and they were compensated for their time.

\subsection{Preliminary Clinical Trial Results}

The study is ongoing. A total of thirty-two women have been recruited to date, out of a target of 60 , with twenty randomized into the agent group. Participants to date are aged 19 to 40 (mean 31.4 ), are $75.9 \%$ white, $75.9 \%$ are currently married, and $72.4 \%$ have high levels of computer literacy.

Eighteen of the intervention participants completed the prenatal module of the system, rating their satisfaction with agent moderately high (average 5.2 on a composite scale of $1=$ negatively satisfied to $7=$ positively satisfied) and attitude towards the agent above neutral (average 4.1 on a composite scale of $1=$ negative attitude to $7=$ positive attitude).

Seven of the intervention participants have already given birth, and have interacted with the agent in the labor and delivery floor of the hospital, and are currently using the postnatal module at home. These seven participants have had a total of 124 (range 4 to 31 each) conversations with the agent in the postnatal system thus far. 


\section{Conclusions and Future Work}

Preliminary results from the pilot study and the clinical trial in progress are promising, and use of the perinatal module has been very high giving us confidence that the breastfeeding promotion agent can have a real impact on postnatal breastfeeding practice.

One of the challenges of breastfeeding is that the needs of the baby, the needs of the mother, and the circumstances of the mother-baby dyad change substantially from birth to six months. These needs also vary significantly from one dyad to another. Hence, the capability for the agent to adapt and adjust the content and nature of its counseling is one of the distinguishing features of this system. The creation of the mandatory topics, suggested topics, and a library of topics also enables more personalized breastfeeding promotion.

It is also important for continuity of education and care to have an ongoing connection with healthcare providers; this system enables that through participant log-in data submissions and recommendations to contact a provider based on the user's responses. The agent's non-judgmental style can be especially effective for helping mothers feel comfortable about reporting their feelings as a new mother.

Because breastfeeding involves health, medical, and non-medical aspects, our system provides a cohesive way to address a range of topics and direct the mother to appropriate sources of assistance as well.

Despite the very complex nature of the longitudinal intervention we designed, the virtual agent provides a persistent, continuous presence across the disparate aspects of the system. Bringing a new baby home can be very stressful, and mothers can have significant anxiety about breastfeeding for the first time. Many new mothers are not sure if they are breastfeeding correctly or whether their baby is getting enough to eat, since they cannot see the milk consumed. We hope the virtual agent provides a sense of stability, reliability and comfort during this stressful but exciting time.

Acknowledgments. Thanks to Juan Fernandez and other members of the Relational Agents Group at Northeastern for their assistance in developing the system. This work is supported by grants from the Hood Foundation and Northeastern University.

\section{References}

1. McInnes, R., Chambers, J.: Supporting breastfeeding mothers: Qualitative synthesis. Journal of Advanced Nursing 62, 407-427 (2008)

2. Saultz, J., Lochner, J.: Interpersonal Continuity of Care and Care Outcomes: A Critical Review. Ann Family Medicine 3, 159-166 (2005)

3. Section on Breastfeeding: Breastfeeding and the use of human milk. Pediatrics 129, e827e841 (2012)

4. http://www.cdc.gov/breastfeeding/data/NIS_data/index.htm 
5. Bickmore, T., Silliman, R., Nelson, K., Cheng, D., Winter, M., Henaulat, L., PaascheOrlow, M.: A Randomized Controlled Trial of an Automated Exercise Coach for Older Adults. Journal of the American Geriatrics Society 61, 1676-1683 (2013)

6. Bickmore, T., Schulman, D., Sidner, C.: Automated Interventions for Multiple Health Behaviors Using Conversational Agents. Patient Education and Counseling 92, 142-148 (2013)

7. Bickmore, T., Puskar, K., Schlenk, E., Pfeifer, L., Sereika, S.: Maintaining Reality: Relational Agents for Antipsychotic Medication Adherence. Interacting with Computers 22, 276-288 (2010)

8. Leite, I.: Using adaptive empathic responses to improve long-term interaction with social robots. User Modeling, Adaption and Personalization 446-449 (2011)

9. Haroon, S., Das, J., Bhutta, Z.: Breastfeeding promotion interventions and breastfeeding practices: a systematic review. BMC Public Health 13, S20 (2013)

10. Labarère, J., Gelbert-Baudino, N., Laborde, L., Arragain, D., Schelstraete, C., François, P.: CD-ROM-based program for breastfeeding mothers. Matern Child Nutr 7, 263-272 (2011)

11. Giglia, R., Binns, C.: The Effectiveness of the Internet in Improving Breastfeeding Outcomes: A Systematic Review. J Hum Lact (2014)

12. Joshi, A., Wilhelm, S., Aguirre, T., Trout, K., Amadi, C.: An Interactive, Bilingual Touch Screen Program to Promote Breastfeeding Among Hispanic Rural Women: Usability Study. JMIR Research Protocols 2, e47 (2013)

13. http://surf-it.soe.ucsc.edu/node/153

14. Bickmore, T., Schulman, D., Shaw, G.: DTask \& LiteBody: Open Source, Standards-based Tools for Building Web-deployed Embodied Conversational Agents. Intelligent Virtual Agents, Amsterdam, Netherlands (2009)

15. Cassell, J., Vilhjálmsson, H., Bickmore, T.: BEAT: The Behavior Expression Animation Toolkit. In: Conference BEAT: The Behavior Expression Animation Toolkit, pp. 477-486. (2004)

16. Bickmore, T., Picard, R.: Establishing and Maintaining Long-Term Human-Computer Relationships. ACM Transactions on Computer Human Interaction 12, 293-327 (2005)

17. Miller, W., Rollnick, S.: Motivational Interviewing: Preparing People for Change. Guilford Press, New York (2002)

18. Bickmore, T., Gruber, A., Picard, R.: Establishing the computer-patient working alliance in automated health behavior change interventions. Patient Educ Couns 59, 21 -30 (2005)

19. Edwards, R. A., Bickmore, T., Jenkins, L., Foley, M., \& Manjourides, J.: Use of an Interactive Computer Agent to Support Breastfeeding. Maternal and child health journal, 17(10), 1961-1968 (2013)

20. Dennis, C., Faux, S.: Development and psychometric testing of the Breastfeeding SelfEfficacy Scale. Research in Nursing Health 22, 399-409 (1999)

21. Horvath, A., Greenberg, L.: Development and Validation of the Working Alliance Inventory. Journal of Counseling Psychology 36, 223-233 (1989)

22. US Department of Health and Human Services. The Surgeon General's call to action to support breastfeeding (2011)

23. Bickmore, T., Schulman, D., \& Yin, L.: Maintaining Engagement in Long-term Interventions with Relational Agents. International Journal of Applied Artificial Intelligence, 24(6), 648-666 (2010)

24. K. L. Koay, D. S. Syrdal, M. L. Walters, and K. Dautenhahn.: A user study on visualization of agent migration between two companion robots. HumanComputer Interaction 13 (2009) 